\title{
Fault Reconstruction Using Sliding Mode Observers
}

\author{
${ }^{1}$ K.Hakiki, ${ }^{3}$ B. Mazari, ${ }^{2}$ A. Liazid and ${ }^{1}$ S. Djaber \\ ${ }^{1}$ Laboratoire d'Automatique et d'Analyse des Systèmes (L.A.AS) Dpt du Génie \\ Electrique ENSET Oran-BP.1523 El'Mnaouar-Oran-Algérie \\ ${ }^{2}$ Laboratoire LTE, ENSET BP.1523 El'Mnaouar-Oran-Algérie \\ ${ }^{3}$ Laboratoire (LDEE), USTOMB-Oran
}

\begin{abstract}
In this study, a nonlinear sliding observer is proposed to explicitly reconstruct fault signals. The novelty lies in the application of the equivalent output injection concept. Previous work in the area of fault detection has used disruption of the sliding motion to detect faults. The aim of the present study was to design a robust observer that slides in the presence of faults based on the response of the estimated outputs of the system. A numerical example of the application to an inverted pendulum mounted on a cart is provided to demonstrate the approach.
\end{abstract}

Key words: Fault diagnosis, sliding mode, observer

\section{INTRODUCTION}

Fundamental purpose of a Fault Detection and Diagnosis (FDD) scheme is to generate an alarm when a fault occurs in the system being monitored and also to identify the nature/location of the fault. In the modelbased monitoring approach, the alarm is triggered when the actual process behavior deviates from its expected behavior as described by its model. The first step of the FDI procedure is to design a set of signals - so-called residuals - that reflect this discrepancy. State observers $^{[1-8]}$, detection filters ${ }^{[9,10]}$, Analytic Redundancy Relations (ARR $)^{[4,7,11]}$ and parameter identification techniques $^{[12-14]}$ are used to generate these residuals. In absence of faults, residuals are designed to be small; once a fault occurs, a subset of residuals are intended to react by becoming greater than some predefined threshold. In the presence of measurement noise and/or perturbation, the effect of the fault on the residual may be masked. A statistical decision method $^{[15]}$ is then applied to trigger an alarm as quickly as possible with minimum errors of false alarms or missed detections. The obtained alarm vector - the socalled fault signature allows to determine which component (or set of components) is faulty. This isolation will enable efficient management of maintenance. In some cases, it is necessary to keep up the system working even in the presence of faults. The control must then be adapted in order to continue to fulfill the system mission or to lead the system to a secure behavioral mode. In that way, one speaks about Fault Tolerant Control ${ }^{[16,17]}$. This tolerant control may be passive or active. In the first case, faults are considered as disturbances and the problem amounts to a classical problem of robust control. In the second case the control is designed such that it automatically adapts to the fault when it occurs. Consequently, the latter scheme makes direct use of the failure detection and diagnosis (FDD) module results. More precisely, it requires accurate knowledge of the nature and features of the fault. This study deals with this particular point and more precisely aims at using sliding mode observers to carry out fault identification.

The concept of a sliding mode emerged from the Soviet Union in the late sixties ${ }^{[18]}$ where the effects of introducing discontinuous control action into dynamical systems were explored. The purpose of the switching control law was to drive the nonlinear plant's state trajectory onto a pre-specified (user-chosen) surface in the state space and to maintain it on this surface for all subsequent time. The resulting reduced-order motion referred to as the sliding motion- was shown to be insensitive to any uncertainty or external disturbance signals which were implicit in the input channels. Thus, based on control theory and because observability is the dual problem of controllability, this inherent robustness property has subsequently been employed in other situations including the problem of state estimation via a discontinuous observer ${ }^{[19-21]}$. Walcott and $\mathrm{Zak}^{[22]}$ use a Lyapunov-based approach to formulate an observer which, under appropriate assumptions, exhibits asymptotic state error decay in the presence of bounded nonlinearities/uncertainties in the input channel. Edwards and Spurgeon ${ }^{[23]}$ propose an observer strategy, similar in style to that of Walcott et Zak, which circumvents the use of symbolic manipulation and offers an explicit design algorithm. Sliding modes have been previously used for fault detection. Sreedhar et $a l .{ }^{[25]}$ consider a model-based sliding observer approach although in their design procedure it is assumed that the states of the system are available. A different approach is adopted by Hermans et Zarrop ${ }^{[26]}$ who attempt to

Corresponding Author: K.Hakiki, Laboratoire d'Automatique et d'Analyse des Systèmes (L.A.AS) Dpt du Génie Electrique ENSET Oran-BP.1523 El'Mnaouar-Oran-Algérie 
design an observer in such a way that in the presence of fault the sliding motion is destroyed. Under appropriate geometric conditions and by transforming a nonlinear system into two different uncertain subsystems, Jiang et $a l .{ }^{[26]}$ designed a sliding mode observer that allows to estimate the faults.

The present study considers the situation when the system states are not all available and provides some new developments in the use of sliding mode observer theory for decoupling the effects of fault signals from the response of the system estimated outputs. The observer is designed to maintain a sliding motion even in the presence of faults which are detected by analyzing the so-called equivalent output injection. The novelty lies in the manipulation of the latter signal to explicitly reconstruct actuator and sensor fault signals.

The proposed method is illustrated on a numerical example: a nonlinear inverted pendulum problem. In addition to the nonlinear dynamics, this example has Coulomb friction in the cart bearings with the result that the cart and the pendulum have a limit cycle oscillation, providing further uncertainty and a challenge to the robust fault estimation problem. The nonlinearities and friction are not considered in the design of the sliding mode observer.

Throughout this study the notation \|\| will be used to represent the Euclidian norm for vectors and (the induced) spectral norm for matrices.

Design of a discountinuous observer: This section introduces the preliminaries and background ideas necessary for the work presented later in the study Canonical Form: Consider the dynamical system

$\dot{x}(t)=A x(t)+B u(t)+D \xi(t, x, u)$

$y(t)=C x(t)$

where $\mathrm{A} \in \mathfrak{R}^{\mathrm{nxn}}, \mathrm{B} \in \mathfrak{R}^{\mathrm{nxm}}, \mathrm{C} \in \mathfrak{R}^{\mathrm{pxn}}, \mathrm{D} \in \mathfrak{R}^{\mathrm{nxq}}$ with $\mathrm{q} \leq \mathrm{p}<$ n.

The triple $(\mathrm{A}, \mathrm{B}, \mathrm{C})$ represents the linear part of the uncertain system given in (1)-(2).

Nonlinear function $\xi: \Re_{+} \times \Re^{\mathrm{n}} \times \mathfrak{R}^{\mathrm{m}} \rightarrow \mathfrak{R}^{\mathrm{q}}$ represents the uncertainties of the model. This function is unknown but bounded so that

$\|\xi(t, x, u)\| \leq r_{l}\|u\|+\alpha(t, y)$

where $r_{1}$ and $\alpha: \Re_{+} \times \Re^{\mathrm{p}} \rightarrow \mathfrak{R}_{+}$are known

Assume that the matrices $\mathrm{B}, \mathrm{C}$ and $\mathrm{D}$ are of full rank and that $(\mathrm{A}, \mathrm{C})$ is observable.

Assumption 1: Suppose that the triple (A,D,C) with $\mathrm{p}>\mathrm{q}$ satisfies the following two conditions

$* \quad \operatorname{rank}(\mathrm{CD})=\mathrm{q}$

* any invariant zero of $(\mathrm{A}, \mathrm{D}, \mathrm{C}) \in \mathrm{C}$. where $\mathrm{C}$. is the open left-half complex plane.

Remark 1: The rank restriction is imposed in order to guarantee the existence of a unique equivalent control of the reduced-order sliding motion for the discontinuous observer that will be defined in the following; the second condition ensures the stabilizability of this observer despite the presence of uncertainty.

\section{Theorem 1:[28]}

Under assumption 1 there exists a linear transformation $\mathrm{z}=\left(\mathrm{z}_{1}^{\mathrm{T}} \mathrm{z}_{2}^{\mathrm{T}}\right)^{\mathrm{T}}=\mathrm{T}_{0} \mathrm{X}$

$\mathrm{T}_{0} \in \mathfrak{R}^{\mathrm{nxn}}$ is a non singular matrix

such that system (1-2) can be rewritten in the following canonical form :

$\left\{\begin{array}{l}\dot{z}_{1}(t)=H_{11} z_{1}(t)+H_{12} z_{2}(t)+B_{1} u(t) \\ \dot{z}_{2}(t)=H_{21} z_{1}(t)+H_{22} z_{2}(t)+B_{2} u(t)+d_{2} \xi(t, z, u) \\ y(t)=z_{2}\end{array}\right.$

where $\mathrm{z}_{1} \in \mathfrak{R}^{(\mathrm{n}-\mathrm{p})}, \mathrm{z}_{2} \in \mathfrak{R}^{\mathrm{p}}$ and matrix $\mathrm{H}_{11}$ is stable.

Proof :[28]

Sliding mode observer: Consider the dynamical system of the form

$$
\left\{\begin{aligned}
\dot{\vec{z}}_{1}(t)= & H_{11} \hat{z}_{1}(t)+H_{12} \hat{y}(t)+B_{1} u(t)-H_{12} e{ }^{(t)} \\
\dot{z}_{2}(t)= & H_{21} \hat{z}_{1}(t)+H_{22} \hat{y}(t)+B_{2} u(t) \\
& -\left(H_{22}-H_{22}^{s}\right) e_{y}(t)+v \\
\hat{y}=\hat{z}_{2} &
\end{aligned}\right.
$$

where $H_{22}^{s}$ is a stable design matrix, $e_{y}=\hat{y}-y$ and the discontinuous vector $v$ is defined by ${ }^{[27]}$ :

$v= \begin{cases}-\rho(t, y, u)\left\|d_{2}\right\| \frac{P_{2} e_{y}}{\left\|P_{2} e_{y}\right\|} & \text { if } e_{y} \neq 0 \\ 0 & \text { otherwise }\end{cases}$

where $\mathrm{P}_{2} \in \Re^{\mathrm{p} \times \mathrm{p}}$ is the symmetric positive definite Lyapunov matrix for $H_{22}^{s}$. The scalar function $\rho: \Re_{+} \times$ $\mathfrak{R}^{\mathrm{p}} \times \mathfrak{R}^{\mathrm{m}} \rightarrow \mathfrak{R}_{+}$

satisfies

$\rho(t, y, u) \geq r_{1}\|u\|+\alpha(t, y)+\gamma_{0}$

and $\gamma_{0}$ is a positive scalar.

If the state estimation errors are defined as $e_{1}=\hat{z}_{1}-z_{1}$ and $e_{2}=\hat{z}_{2}-z_{2}$ then it is straightforward to obtain

$\dot{e}_{1}(t)=H_{11} e_{1}(t)$

$\dot{e}_{y}(t)=H_{21} e_{1}(t)+H_{22}^{s} e_{y}(t)+v-d_{2} \xi$

since $e_{y}=e_{2}$

\section{Theorem 2:[23]}

The nonlinear error system in (8)-(9) is quadratically stable and a sliding motion takes place forcing $\mathrm{e}_{\mathrm{y}}=0$ in finite time. As a consequence, system (5) is a robust sliding mode observer for system (4).

Proof:[23]. 
In the original coordinates, the robust observer for the system (1-2) can conveniently be written as

$\dot{\hat{x}}(t)=A \hat{x}(t)+B u(t)-G_{l} e_{y}(t)+G_{n} v$

$\hat{y}(t)=C \hat{x}(t)$

where the linear and the nonlinear gains are respectively

$$
G_{l}=T_{0}^{-1}\left[\begin{array}{c}
H_{12} \\
H_{22}-H_{22}^{s}
\end{array}\right] \quad \text { and } G_{n}=T_{0}^{-l}\left[\begin{array}{c}
0 \\
I_{p}
\end{array}\right]
$$

Remark 2: In the case where $\mathrm{p}=\mathrm{q}$, an observer of the form (10) which is insensitive to the uncertainty (3) will exist if and only if $\operatorname{det}(C D) \neq 0$ and invariant zeros of $(A, D, C) \in C$. That is, the triple $(A, D, C)$ is minimum phase and relative degree 1 and the sliding motion is totally determined by these ( $n-p)$ invariant zeros.

Remark 3: Consider the hyperplane given by $\mathrm{S}_{0}$ $=\left\{\mathrm{e} \in \mathfrak{R}^{\mathrm{n}}: \mathrm{Ce}=0\right\}$

where $e=\hat{x}-x$. Even in the special case where $\mathrm{D}=\mathrm{B}$, the Walcott-Zak observer formulation ${ }^{[22]}$ is different from that of (10) for $\mathrm{p}>\mathrm{m}$ since their results guarantee sliding will take place on the surface in the error space defined by $S_{w z}=\left\{e \in \Re^{n}: F C e=0\right\}$ for a certain matrix $\mathrm{F} \in \mathfrak{R}^{\mathrm{m} \times \mathrm{p}}$. This does not imply $\mathrm{Ce}=0$, since the null space of $\mathrm{F}$ is not empty; and therefore, the Walcott-Zak's observer, differently from (10), does not necessarily track the system outputs perfectly.

\section{RECONSTRUCTION OF FAULTS}

Problem statement: This section considers the use of the sliding mode observer defined in section II to reconstruct actuator and sensor faults.

Consider a linear system subject to certain faults

$x(t)=A x(t)+B u(t)+D f_{a}(t)$

$\mathrm{y}(\mathrm{t})=\mathrm{Cx}+\mathrm{f}_{\mathrm{s}}(\mathrm{t})$

where the dimensions and ranks of matrices have been indicated in section II. Nonlinear functions $\mathrm{f}_{\mathrm{a}}(\mathrm{t})$ and $\mathrm{f}_{\mathrm{s}}(\mathrm{t})$ are deemed to represent actuator and sensor faults respectively and are assumed to be bounded.

The objective is to synthesize an observer to generate a state estimate $\hat{x}(t)$ and output estimate $\hat{y}=C \hat{x}$ such that a sliding mode is established in which $e_{y}(t)=\hat{y}(t)-y(t)$ is forced to zero in finite time, even in the presence of faults. It will be shown that provided a sliding motion will be attained, estimates of $f_{a}(t)$ and $f_{s}(t)$ can be computed by approximating the socalled equivalent output injection signal required to maintain sliding motion.

Reconstruction of the Actuator Fault Signals

Consider initially the case when $\mathrm{f}_{\mathrm{a}}(\mathrm{t}) \neq 0$ and $\mathrm{f}_{\mathrm{s}}(\mathrm{t})=$ 0 During the sliding motion, $\mathrm{e}_{\mathrm{y}}=0$ and $\dot{e}_{y}=0$. In the coordinates of the canonical form in Section II.A, equation (9) becomes

$$
0=H_{2 l} e_{1}(t)-d_{2} f_{a}(t)+v_{e q}
$$

where $v_{\mathrm{eq}}$ is the equivalent control. From Eq. (8), it follows that $\mathrm{e}_{1} \rightarrow 0$ and therefore

$v_{\text {eq }} \rightarrow \mathrm{d}_{2} \mathrm{f}_{\mathrm{a}}(\mathrm{t})$

The equivalent control may be recovered by using a low pass filter ${ }^{[21]}$. Here, an alternative approach will be employed: Suppose, instead of the discontinuous component in (6), that we use the continuous approximation

$v_{\delta}=-\rho\left\|d_{2}\right\| \frac{P_{2} e_{y}}{\left\|P_{2} e_{y}\right\|+\delta}$

where $\delta$ is a small positive scalar.

The equivalent control can be approximated by (15) to any required accuracy by a small enough chosen value of $\delta$. Since $\operatorname{rank}\left(\mathrm{d}_{2}\right)=\mathrm{q}$, it follows from Eq. (14) that

$$
f_{i}(t) \approx-\rho\left\|d_{2}\right\|\left(d_{2}^{T} d_{2}\right)^{-1} d_{2}^{T} \frac{P_{2} e_{y}(t)}{\left\|P_{2} e_{y}(t)\right\|+\delta}
$$

The key point is that the signal on the right-hand side of (16) can be computed on-line and depends only on the output estimation error $\mathrm{e}_{\mathrm{y}}$.

Sensor faults detection and diagnosis: Now consider the case when $\mathrm{f}_{\mathrm{a}}=0$ and consider the effect of $\mathrm{f}_{\mathrm{s}}(\mathrm{t})$.

In this situation, from Eq. (13) it follows that

$e_{y}(t)=C e(t)-f_{s}(t)$

The dynamics of the state estimation error (8-9) is given by

$\dot{e}_{1}(t)=H_{11} e_{1}(t)+H_{12} f_{s}(t)(17)$

$\dot{e}_{y}(t)=H_{21} e_{1}(t)+H_{22}^{s} e_{y}(t)-\dot{f}_{s}(t)+H_{22} f_{s}(t)+v(18)$

Note that $\mathrm{f}_{\mathrm{s}}$ and $\dot{f}_{s}$ appear as output disturbances and thus $\rho$ in Eq. (6) must be chosen to be sufficiently large to maintain sliding in the presence of these disturbances. Arguing as before, provided a sliding motion is attained, Eq.(18) can be written as

$0=H_{2 l} e_{1}-\dot{f}_{o}(t)+H_{22} f_{s}(t)+v_{e q}$

Thus for slowly varying faults-and if the dynamics of the sliding motion are sufficiently fast,

$v_{e q}=-\left(H_{22}-H_{2 I} H_{11}^{-1} H_{12}\right) f_{S}(19)$

As previously, $v_{\text {eq }}$ can be calculated from Eq. (15); and consequently, if $\left(H_{22}-H_{2 I} H_{11}^{-1} H_{12}\right)$ is nonsingular, the fault signal can be obtained from Eq. (19).

Note that from the Schur expansion $\operatorname{det}(\mathrm{H})=\operatorname{det}\left(\mathrm{H}_{11}\right) \cdot \operatorname{det}\left(\mathrm{H}_{22}-\mathrm{H}_{21} \mathrm{H}_{11}{ }^{-1} \mathrm{H}_{12}\right)$ and thus $\left(H_{22}-H_{21} H_{11}^{-1} H_{12}\right)$ is nonsingular if and only if $\operatorname{det}(\mathrm{H}) \neq 0$. 


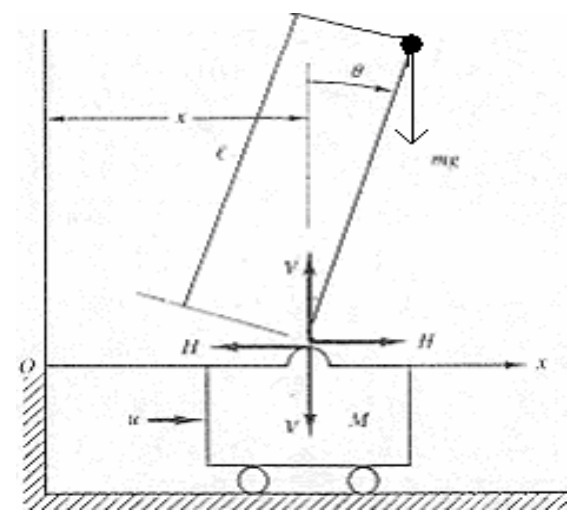

Fig. 1: Schematic of inverted pendulum

Table 1: Model parameters for the inverted pendulum

\begin{tabular}{llllll}
\hline $\mathrm{M}$ & $(\mathrm{kg})$ & 3.2 & $\mathrm{~F}_{\mathrm{x}}$ & $(\mathrm{kg} / \mathrm{sec})$ & 6.2 \\
$\mathrm{M}$ & $(\mathrm{kg})$ & 0.535 & $\mathrm{~F}_{\theta}$ & $\left(\mathrm{kg} / \mathrm{m}^{2}\right)$ & 0.009 \\
$\mathrm{~J}$ & $\left(\mathrm{kgm}^{2}\right)$ & 0.062 & $\mathrm{~g}$ & $\left(\mathrm{~m} / \mathrm{sec}^{2}\right)$ & 9.807 \\
$\mathrm{~L}$ & $(\mathrm{~m})$ & 0.365 & & & \\
\hline
\end{tabular}

Remark 4: Note that even if $\left(H_{22}-H_{21} H_{11}^{-1} H_{12}\right)$ is singular, it may still be possible to reconstruct some of the sensor faults $f_{s}$ depending on the structure of the rank deficiency, as it will be shown in the next section.

Remark 5: The fault detection approach adopted here is based on the equivalent control concept and thus does not interact in any way with the use of the sliding mode observer as a state estimator. A set of states compatible with the current plant output will always be produced; this is not the case with those fault detection methods ${ }^{[25]}$ which rely on breaking the sliding motion in order to detect faults.

Numerical example: To illustrate the method, consider the inverted pendulum mounted on a cart shown in Fig. 1 , under the dynamic output feedback control developed in $^{[29]}$. The equations of motion of the system are

$(M+m) \ddot{x}+F_{x} \dot{x}+m l\left(\ddot{\theta} \cos \theta-\dot{\theta}^{2} \sin \theta\right)=u$

$J \ddot{\theta}+F_{\theta} \dot{\theta}-m \lg \sin \theta+m l \ddot{x} \cos \theta=0$

where the particular values of the system parameters are given in Table 1.

Suppose that the state variables $\mathrm{x}, \theta$ and $\dot{x}$ are measured. The control law which requires the state $\dot{\theta}$ to be reconstructed, does not have to be influenced by faults.

A linearization of the nonlinear equations around the equilibrium point at the origin generates the system triple

$$
A=\left[\begin{array}{cccc}
0 & 0 & 1 & 0 \\
0 & 0 & 0 & 1 \\
0 & -1.9333 & -1.9872 & 0.0091 \\
0 & 36.9771 & 6.2589 & -0.1738
\end{array}\right]
$$

$$
B=\left[\begin{array}{c}
0 \\
0 \\
0.3205 \\
-1.0095
\end{array}\right] \quad C=\left[\begin{array}{llll}
1 & 0 & 0 & 0 \\
0 & 1 & 0 & 0 \\
0 & 0 & 1 & 0
\end{array}\right]
$$

In this particular situation, any actuator faults, will occur in the input channel and hence in the notation of section II the fault distribution matrix $\mathrm{D}=\mathrm{B}$. It can be readily established that the existence conditions of $\mathrm{T}_{0}$ are satisfied and using the algorithm that results from these conditions, it can be shown that the linear change of coordinates $\mathrm{T}_{\mathrm{o}}$ is

$T_{o}=\left[\begin{array}{cccc}0 & -9.8549 & 3.1498 & 1.000 \\ 1.000 & 0 & 0 & 0 \\ 0 & 1.000 & 0 & 0 \\ 0 & 0 & 1.000 & 0\end{array}\right]$

and that the system (20) may be transformed into the canonical form (4) where

$\mathrm{H}=\left[\begin{array}{ll}\mathrm{H}_{11} & \mathrm{H}_{12} \\ \mathrm{H}_{21} & \mathrm{H}_{22}\end{array}\right]=\mathrm{T}_{\mathrm{O}} \mathrm{AT}_{\mathrm{O}}^{-1}=$

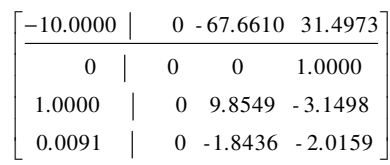

$\mathrm{d}_{2}=0.3205$ and

$c=\left[\begin{array}{l|lll}0 & 1 & 0 & 0 \\ 0 & 0 & 1 & 0 \\ 0 & 0 & 0 & 1\end{array}\right]$

where by design $\mathrm{H}_{11}=-10$. In this particular case

$H_{22}^{s}=\operatorname{diag}(-11,-12,-13)$ which furnishes the linear component of the observer with poles approximately three times faster than the closed-loop poles of the controlled plant. The symmetric positive-definite matrix $\mathrm{P}_{2}$ has been selected as the unique solution of the Lyapunov equation

$P_{2} H_{22}+H_{22}^{T} P_{2}=-I$

In this particular design the scalar function $\rho=75$ and the observer design is complete.

Estimation of faults: It can be verified that the eigenvalues of the matrix $A$ are

$\{0,5.8702,-6.3965,-1.6347\}$ and thus the steady-state gain from $\mathrm{f}_{\mathrm{s}}$ to $v_{\mathrm{eq}}$ is singular. It can be verified that

$\left(H_{22}-H_{21} H_{11}^{-1} H_{12}\right)=\left[\begin{array}{ccc}0 & 0 & 1 \\ 0 & 3.0888 & 0 \\ 0 & -1.9052 & -1.9872\end{array}\right]$

is clearly rank deficient. However, if $v_{\mathrm{eq}, \mathrm{i}}$ and $\mathrm{f}_{\mathrm{s}, \mathrm{i}}$ represent the ith components of the vectors $v_{e q}$ and $f_{s}$ and from Eq.(19) and Eq.(22), it is apparent that

$v_{e q, 1} \approx-f_{s ., 3}$

$v_{e q .2} \approx-3.0888 f_{s, 2}$

It is also clear that any fault in the first output channel has no direct long-term effect on $v_{\text {eq }}$. Furthermore, 
because of the structure of $d_{2}$ in (21), it can be verified that

$\left(d_{2}^{T} d_{2}\right)^{-1} d_{2}^{T}=\left[\begin{array}{lll}0 & 0 & 3.1200\end{array}\right]$

and so from (14)

$v_{e q, 3} \approx 0.3205 f_{a}$

Thus the three components of the equivalent output injection signal, properly scaled, provide estimate of $\mathrm{f}_{\mathrm{s}, 3}$ and $\mathrm{f}_{\mathrm{s}, 2}$ and $\mathrm{f}_{\mathrm{a}}$ respectively and may be used as detector signals .

\section{SIMULATION RESULTS}

Figure 2 shows the effect of a parabolic fault in the input channel. As predicted, the third detector signal reproduces the fault signal (Eq. (25)) whilst not affecting the other two signals ( Eq. (23-24)).
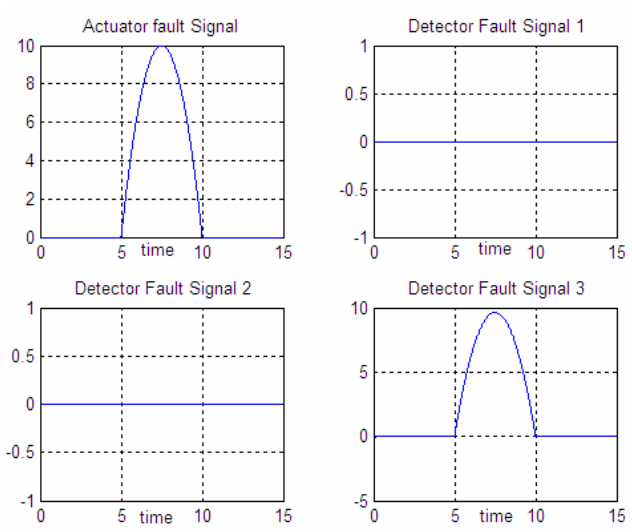

Fig. 2: Effect of a parabolic fault in the input channel
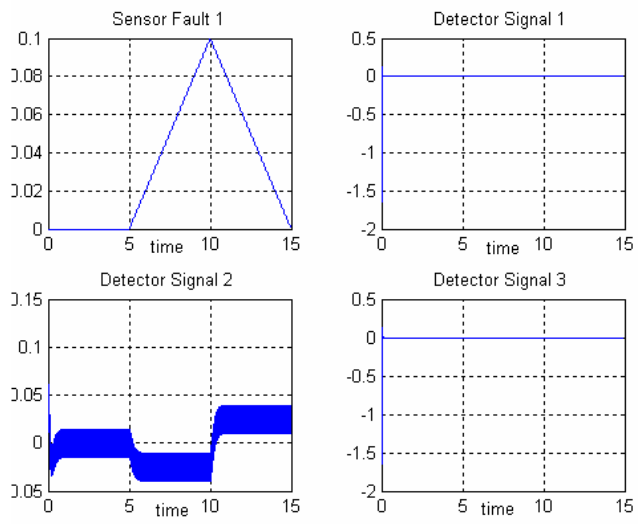

Fig. 3: Effect of a ramp in the first output channel

Figure 3 shows the effect of a ramp in the first output channel. As predicted, the detector signals (Eq. (23-24)) do not reproduce the fault signal (although the second detector signal approximates the gradient of the fault signal).

Figures 4 and 5 show that the appropriate detector signal reproduces the ramp fault signals in output channels 2 and 3 . In both cases the detector signal 3 is influenced as well.
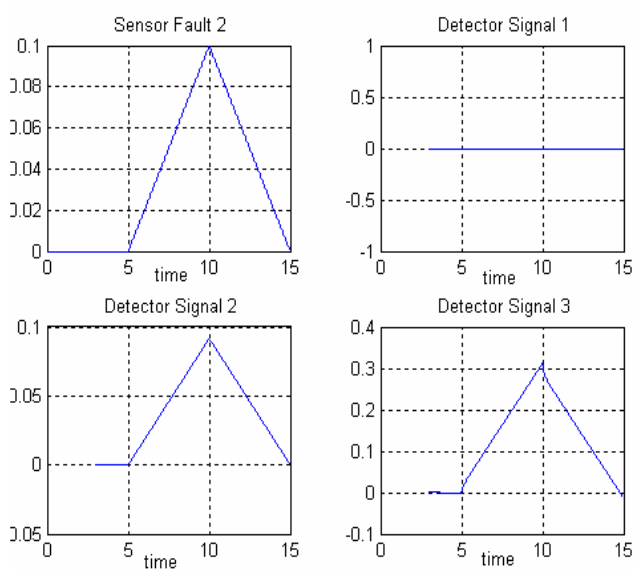

Fig 4: Effect of a ramp in the second output channel
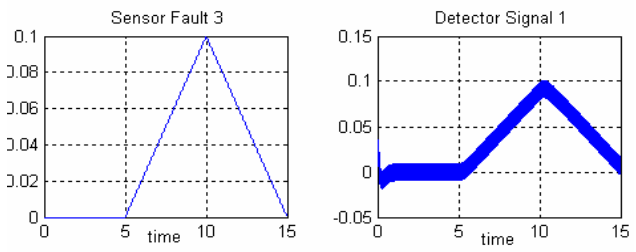

Detector Signal 2
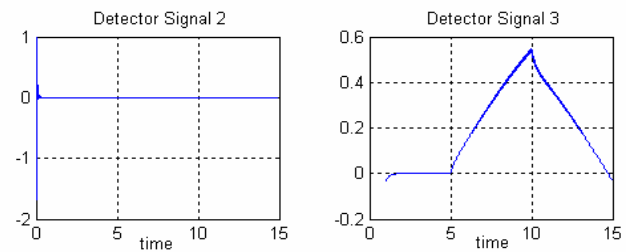

Fig. 5: Effect of a ramp in the third output channel

Remark 6: In the Walcott-Zak approach, the equivalent control would have only one component making it difficult to distinguish between faults in different channels.

\section{CONCLUSION}

This study has explored the use of sliding mode ideas for the purpose of fault detection and diagnosis. The approach adopted here differs significantly from the work of Hermans et Zarrop in that the underlying intention is to ensure that sliding is maintained even in the presence of faults. By examining an equivalent output injection signal it has been demonstrated that certain fault signals can be faithfully reproduced.

The inherent perspectives of this work are situated in the general context of active fault tolerant control for nonlinear systems. The authors' research interest will be particularly to look forward to:

* Extend fault identification to nonlinear systems and especially in the case where a part of the nonlinearity is known (nonlinear function of the state and inputs) and may be used to design the observer

* Develop tolerant sliding mode controls to faults integrating fault signals estimation. 


\section{ACKNOWLEDGEMENTS}

The present research work has been supported by Science and Technology for Safety in Transportation and funded by the European Union, the Délégation Régionale à la Recherche et à la Technologie, the Ministère Délégué à l'Enseignement Supérieur et à la Recherche, the Région Nord Pas de Calais ( France) and the Centre National de la Recherche Scientifique.

\section{REFERENCES}

1. Chen, J., R. Patton and H. Zhang, 1996. Design of unknown input observers and robust fault detection filters. Intl. J. Control, 63: 85-105.

2. Ding, X. and P. Frank, 1990. Fault detection via factorization approach. Systems and Control Lett., 14: $431-436$.

3. Gertler, J., 1988. Survey of model-based failure detection and isolation in complex plant. IEEE Control Systems Mag., 3: 3-11.

4. Gertler, J., 1991. Analytical redundancy methods in fault detection and isolation. Proc. IFAC/IMACS Symposium, Safeprocess '91, Baden-Baden, pp: 9-21.

5. Hou, M. and P. MuKller, 1992. Design of observers for linear systems with unknown inputs. IEEE Trans. Automatic Control, AC-37: 871-875.

6. Hou, M. and P. MuKller, 1994. Fault detection and solution observers. Intl. J. Control, 60: 827- 846.

7. Patton, R. and J. Chen, 1991. Robust fault detection using eigenstructure assignment: A tutorial consideration and some new results. Proc. 30th IEEE Conf. Decision and Control, pp: 22422247, Brighton.

8. Patton, R. and J. Chen, 1997. Observer-based fault detection and isolation: Robustness and applications. IFAC J. Control Engg. Pract., 5: 671682.

9. Ge, W. and Z. Fang, 1988. Detection of faulty components via robust observation. Intl. J. Control, 47: 581-599.

10. Massoumnia, M., 1986. A Geometric approach to failure detection and identification in linear systems. Ph. D. Thesis, Cambridge, MIT.

11. Staroswiecki, M., J.P. Cassar and V. Cocquempot, 1993. Generation of optimal structured residuals in the parity space. 12th IFAC World Congress, Sidney, Australia, Jul. 18-23.

12. Isermann, R., 1984. Process fault diagnosis based on modelling and estimation methods-A survey. Automatica, 113: 387-404.

13. Isermann, R. and B. Freyermuth, 1991. Process fault diagnosis based on process model knowledgePart I: Principles for fault diagnosis with parameter estimation. Trans. ASME, 113: 620- 626.

14. Sprösser, Th. and G.L. Gissinger, 1994. Identification based sensor fault detection and localization. Application to an inverted pendulum. IFAC / IMACS Symposium SAFEPROCESS ' 94, Helsinki.
15. Basseville, M. and I. Nikiforov, 1993. Detection of abrupt changes- Theory and applications. Prentice Hall Information and System Science Serie (T.Kailath, ed.) Englewood Cliffs.

16. Blanke, M., M. Kinnaert, J. Lunze and M. Staroswiecki, 2003. Diagnosis and fault tolerant. Springer Verlag Berlin Heidelberg.

17. Jiang, B., M. Staroswiecki and V. Cocquempot, 2003. Active fault tolerant control for a class of nonlinear systems. Proc. of IFAC Safeprocess' 03, Washington, Control. Springer-Verlag.

18. Emelyanov, S.V., 1967. Variable Structure Control Systems. Moscow-Nauka. 1967 (in Russian); also Munchen-Wien: Oklenburg Verlag. 1969 (in German).

19. Drakunov, S. and V. Utkin, 1995. Sliding mode observers: Tutorial. Proc. 34th IEEE Conf on Decision and Control, pp: 3376-3379.

20. Slotine, J., J. Hedrick and E. Misawa, 1987. On sliding observers for nonlinear systems. Trans. ASME: J. Dynamic Systems, Measurement and Control, 109: 245-252.

21. Utkin, V., 1992. Sliding modes in control optimization. Berlin Springer.

22. Walcott, B. and S. Zak, 1988. Combined observercontroller synthesis for uncertain dynamical systems with applications. IEEE Trans. Systems, Man and Cybernetics, 18: 88-104.

23. Edwards, C. and S. Spurgeon, 1994. On the development of discontinuous observers. Intl. J. Control, 59: 1211- 1229.

24. Sreedhar, R., B. Fernandez and G. Masada, 1993. Robust fault detection in nonlinear systems using sliding mode observers. Proc. IEEE Conf. Control Applications, pp: 715-721.

25. Hermans, F. and M. Zarrop, 1996. Sliding mode observers for robust sensor monitoring. Proc. 13th IFAC World Congress, pp: 211-216.

26. Jiang, B., M. Staroswiecki and V. Cocquempot, 2004. Fault estimation in nonlinear uncertain systems using robust/sliding mode observers. IEE Control Theory and Application (IEE-CTA), 1: 29-37.

27. Ryan, E.P. et M. Corless, 1984. Ultimate bondedness and asymptotic stability of a class of uncerutain dynamical systems via continuous and discontinuous control. IMA J. Mathematical Control and Information, 1: 223-242.

28. Edwards, C. and S. Spurgeon. Sliding Mode Control theory and applications. Taylors and Francis.

29. Hakiki, K., V. Cocquempot, B. Mazari and S. Djaber, 2005. Dynamic output feedback sliding mode control for a class of uncertain systems using an observer based compensator. Proc. 3rd Intl. Conf. Electrotechnics, ICEL'2005’USTO MB. 